\title{
Encuentro y Vinculación Afectiva: Pilotaje y Proceso de Nutrición Relacional en Educación
}

\section{Personal Encounter and Affective Links: "Pilot Guidance" and the Relational Nurturing Process in Education}

\author{
Antonio Vicente Vaquer Chiva, Virginia Elena Carrero Planes y Francisco Juan García Bacete \\ Universitat Jaume I, Spain
}

\begin{abstract}
Resumen. Los centros educativos constituyen el marco preferente de socialización y desarrollo de los adolescentes en España. La preocupación por algunos fenómenos disruptivos que se producen en el marco educativo dirige la atención a investigar los aspectos de la relación educativa que contribuyen de forma positiva al desarrollo y aprendizaje del alumnado. A partir de un diseño de investigación cualitativa, siguiendo los criterios de muestreo teórico propuestos en la metodología de análisis cualitativo del "Grounded Theory", se realizaron veintitrés entrevistas individuales a padres/madres, profesorado y alumnado de educación secundaria. Los resultados obtenidos permiten proponer el "encuentro" entre la persona del alumno y del profesor, con su correspondiente aceptación y reconocimiento mutuo, como factor central que posibilita la vinculación afectiva y el ejercicio del pilotaje entre el profesor y el alumno. La emergencia de la "nutrición relacional" como proceso social básico, implica que el crecimiento personal y educativo se potencia a través del apoyo afectivo y de guía en el aprendizaje. La función del pilotaje educativo, tanto individual como de aula, conforma el centro de la relación educativa y contribuye al desarrollo pleno del alumnado.
\end{abstract}

Palabras clave: adolescencia, Grounded Theory, nutrición relacional, pilotaje, vinculación afectiva.

\begin{abstract}
Educational institutions represent the special setting for teenagers' socialization and development in Spain. The concern for some disruptive phenomenon which take place within the educational setting draws the attention to investigate the aspects of the educational relationship that contribute positively to students' development and learning. From a qualitative research design, according to the criteria of theoretical sampling proposed in the methodology of qualitative data analysis of the «Grounded Theory», twenty-three individual interviews were done to secondary school parents, teachers and students. The achieved results allow to suggest the «encounter» between student and teacher, with its respective acceptance and mutual appreciation, as a central issue that enables affective link and the practice of "pilot guidance". The emergence of the basic social process referred as «relational nurturing» implies that affective support and an instructional guide empower educational and personal growth. The "pilot guidance" function, both with individuals and in class, constitutes the center of the educational relationship fostering the students' full growth.

Keywords: adolescent, affective bonding, Grounded Theory, pilot guidance, relational nurturing.
\end{abstract}

Dado que la aceleración del cambio social se ha convertido en una de las características centrales de nuestro tiempo, las pautas y modelos con los que se encara la educación de los niños y jóvenes han dejado de ser "obvias" y socialmente ajustadas a una visión estática y jerárquica de la sociedad para diversificarse en un abanico complejo de prácticas y concepciones educativas que, no pocas veces, llevan a situaciones de confusión respecto a la forma "correcta" de actuar.

Los roles educativos clásicos se ven puestos en tela de juicio a la par que las nuevas exigencias laborales y

Correspondencia: Antonio V. Vaquer Chiva. Departamento de Psicología Evolutiva, Educativa, Social y Metodología. Facultad de Ciencias Humanas y Sociales. Avda Vicente Sos Baynat s/n. Universitat Jaume I. 12071-Castellón, Spain. E-mail: avaquer@guest.uji.es profesionales obligan a los padres a compartir cada vez más su papel con otras instituciones o servicios. Escuelas e institutos se han convertido en la principal institución social en que se produce la convivencia social como afirma Fernández Enguita (2001).

Naturalmente, el sistema educativo no podía quedar al margen ni de las transformaciones sociales ni de las consecuencias que para la relación educativa acarrea la situación mencionada anteriormente. Prats y Raventós (2005), en la introducción de su libro acerca de los sistemas educativos europeos, se interrogan acerca si estamos ante una crisis o ante una transformación del sistema educativo y afirman: "Ciertamente, la educación europea está viviendo, desde hace décadas, transformaciones constantes que intentan superar los desajustes que se producen tanto en orden interno como externo". 
En nuestro país, los institutos estaban concebidos para actuar de un modo selectivo con y sobre solamente una parte previamente aceptada y adaptada del alumnado (Fernández Enguita, 2001). Desde la aplicación de la LOGSE se ven confrontados al desafío que implica la universalización de la educación en la etapa de la transformación adolescente y dentro de una sociedad que está perdiendo las referencias. El reto que conlleva la escolarización de alumnado extranjero aumenta las probabilidades de fracaso en los inmigrantes del sur (Fernández, Mena y Rivera, 2010) añadiendo la dificultad de adaptación del sistema a la cuestión intercultural.

Las sucesivas reformas y contrarreformas del sistema educativo han hecho más confuso el panorama de la educación y no se ha producido un debate constructivo acerca de las dificultades que como sociedad estamos teniendo para impulsar el desarrollo socializado de la juventud. Los problemas que emergen como consecuencia de todo lo anterior (falta de disciplina, rebeldía, fracaso escolar, problemas de convivencia como el acoso y la violencia, tasas de drogadicción entre jóvenes, agresiones de hijos e hijas a padres... ) son tratados por los medios de comunicación de forma sensacionalista y poco constructiva.

La cuestión de la autoridad en educación ha comenzado a inquietar al mundo educativo de la mano de temas relacionados con la violencia escolar y la desafección de los estudiantes como ha sido puesto de relieve por diversos autores (Cava, Musitu y Murgui, 2006; Marchesi, 2004; Marina, 2006).

Nos encontramos, pues, ante una situación tremendamente compleja que afecta a uno de los sectores más sensibles de nuestra sociedad (niños y adolescentes) y a los centros escolares que, junto con la familia, son los encargados de transmitirles los conocimientos, valores, actitudes y mecanismos que les permitirán desarrollarse como personas, incorporarse a la sociedad y contribuir al enriquecimiento social (en el sentido amplio y noble de la palabra enriquecimiento). El fracaso en dicha tarea puede perjudicar gravemente el funcionamiento social.

En último término la relación educativa descansa en la relación profesor/a-alumno/a y en el aula como marco social en el que actúa dicha relación. Existe un creciente consenso en que la naturaleza y calidad de las relaciones entre los estudiantes y sus profesores juega un papel crítico en la motivación e implicación de los estudiantes de los aprendizajes escolares (Wentzel, 2009). Así pues, ¿Cómo se puede abordar de forma productiva la relación profesor-alumno a pesar de todas las dificultades expuestas anteriormente? ¿Cuáles pueden ser las claves que permitan establecer una relación educativa positiva?

El interés y reflexión derivadas de estas cuestiones focalizan el objetivo del presente estudio en comprender los mecanismos por los que el profesorado y el alumnado establecen una relación educativa que no sólo contempla la ayuda instruccional sino que favorece el desarrollo integral del alumnado.

A partir de estas condiciones previas, y preservando la naturaleza relacional de la pregunta de investigación planteada se ha buscado una metodología de investigación que permitiera interrogar el contexto educativo favoreciendo el descubrimiento de procesos latentes desde un nivel empírico. Las condiciones específicas del estudio requerían el uso de metodologías constructoras de teoría, como la Teoría fundamentada", capaz de dar cuenta de la variabilidad de las condiciones emergentes, a partir de la emergencia de procesos sociales básicos fundamentados en los datos empíricos. La no existencia de marcos o preconcepciones teóricas previas, evita la distorsión de la interpretación de los datos y posibilita un acercamiento a la comprensión de la relación profesor-alumno desde perspectivas hasta ahora poco exploradas en la investigación educativa.

\section{Metodología}

\section{Selección de la Teoría Fundamentada}

Para encarar estas preguntas se requiere de una perspectiva metodológica, constructora-generadora de teoría, que permita abordar la comprensión y explicación de un proceso tan complejo como son las relaciones personales en secundaria y su influencia en el desarrollo personal y escolar del alumnado. Desde aproximaciones cualitativas, se pretende la comprensión del problema en su complejidad, evitando preconcepciones incluyendo la subjetividad de los informantes como un dato que contribuya a la comprensión de la dinámica relacional en el contexto educativo. (Charmaz, 2005). Hemos considerado que la metodología más adecuada para estos fines es la metodología de la Teoría Fundamentada o Grounded Theory propuesta por Glaser y Straus (1967) (GT en adelante), por tratarse de una metodología que permite poner el énfasis tanto en las relaciones del contexto educativo como en la integración de la experiencia subjetiva.

Se trata de una metodología de análisis que tiende al descubrimiento y generación de una teoría fundamentada sobre el área sustantiva de estudio, desde un conjunto de proposiciones teóricas. La utilización del método comparativo constante (MCC) y del muestreo teórico (MT) conforma un diseño emergente donde las fases de obtención y análisis de datos se suceden de modo iterativo. De este modo, la inducción y la posibilidad de generalización se aseguran mediante un procedimiento de comparación sistemática de los nuevos incidentes encontrados con las categorías previamente obtenidas (MCC) y en la aplicación sistemática del muestreo teórico, desde donde las nuevas categorías encontradas guían la elección del foco y la unidad de análisis. La vuelta al campo de estudio cesa una vez se 
ha alcanzado el nivel de saturación teóricas en las categorías encontradas, lo que implica que a pesar de elegir grupos comparativos tan distintos como sea posible, los nuevos datos obtenidos no añaden nada más significativo a las categorías ya encontradas (MT).

\section{Fases de la investigación}

Las fases de investigación contempladas en el estudio han sido:

- Fase de exploración: durante la fase de exploración nos hemos aproximado al campo y se ha delimitado el problema de investigación a partir de entrevistas lo más abiertas posible. En esta fase la codificación ha sido abierta y las categorías todavía poco relacionadas entre sí. En esta fase se focalizó el centro de la investigación en los aspectos afectivos de alumnado, padres y profesores en el proceso educativo.

- Fase de profundización: en la fase de profundización se centró la investigación en la comparación entre categorías y en la codificación teórica. En esta fase la investigación se centra en los aspectos afectivos que inciden de forma positiva en la socialización y aprendizaje del alumnado. Durante esta fase se decide centrarse en los programas educativos del IES Álvaro Falomir por los avances en socialización y aprendizaje que se está constatando con dicho alumnado. Se empieza a producir la jerarquización y conexión entre las categorías aunque todavía hay que trabajar en el proceso de clasificación teórica. El proceso de comparación es ya únicamente entre categorías.

- Fase de saturación: Se busca saturar la categoría central. La comparación es totalmente teórica, se definen las categorías y se especifican las propiedades de las mismas. Al no aparecer datos nuevos, el campo se considera saturado y se deja de recoger datos.

- Fase de clasificación y esquema teórico: En esta fase la comparación es teórica y entre categorías de forma que se estructuran en relación al problema de investigación y emergen las proposiciones teóricas. La estructuración en torno al problema de investigación da lugar al esquema teórico que orientará la escritura teórica.

- Fase de escritura teórica: En esta fase se desarrolla y se plasma el esquema teórico en un informe de investigación para realizar la comunicación de las hipótesis teóricas y de sus implicaciones.

\section{Objetivos}

Hemos mencionado anteriormente que el objetivo del presente estudio es comprender los mecanismos por los que el profesorado y el alumnado establecen una relación educativa que no sólo contempla la ayuda instruccional sino que favorece el desarrollo integral del alumnado. Después del análisis de los primeros datos, y dado el diseño emergente, la investigación se focalizó sobre los aspectos afectivos que posibilitan el desarrollo positivo del adolescente a partir de la comprensión de la influencia ejercida por la relación educativa. Esta focalización de la investigación buscaba profundizar en el conocimiento de los procesos socioafectivos en la relación profesorado-alumnado, y estudiar las condiciones educativas que influyen en el desarrollo socioafectivo del alumnado.

\section{Participantes}

En la fase exploratoria se analizaron datos de seis entrevistas individuales realizadas a familias, alumnos y profesores de Castellón y la fase de profundización se realizó con alumnado, padres y madres y profesorado del Instituto de Enseñanza Secundaria Álvaro Falomir de Almassora (Castellón) (IES Álvaro Falomir). El IES Álvaro Falomir recibe alumnado de cuatro centros de primaria situados en la periferia de Almassora. Dos de los centros de primaria están considerados de Acción Educativa Singular y, en general, la zona de influencia del IES se puede considerar como socialmente desfavorecida y el alumnado presenta un cierto riesgo de fracaso educativo. Dada esta realidad en el IES Álvaro Falomir existe un programa de Compensación Educativa en el que se pretende que el alumnado pueda tener un seguimiento altamente personalizado, que desarrolle las capacidades que se recogen en los objetivos generales de la ESO y que alcance niveles de socialización, madurez y convivencia adecuados a su edad. Algunas características del programa son: la reducción del número de profesorado, la disminución del número de alumnos por aula -un máximo de quince-, la organización didáctica en ámbitos de conocimiento y el refuerzo de la función tutorial. Algunas materias se imparten únicamente para el alumnado del programa mientras que otras se cursan conjuntamente con el alumnado de otro grupo de su nivel -grupo de referencia-. En concreto, en el estudio ha participado alumnado, profesorado y familias del Programas de Adaptación Curricular en Grupo (PACG) y de Diversificación Curricular (PDC) del IES Álvaro Falomir d'Almassora (Castellón). Se eligió el grupo en función de los buenos resultados, tanto académicos como de madurez personal del alumnado, que se estaba obteniendo con los mencionados programas.

En la fase de exploración se realizaron 6 entrevistas abiertas en profundidad ( 2 alumnos, 2 profesores y 2 padres). En esta fase se buscó la mayor diversidad posible de los entrevistados en cuanto a género, rendimiento escolar y nivel educativo en el que se impartía la docencia y experiencia profesional. También se analizaron datos de los medios de comunicación relativos 
a la educación secundaria y de una entrevista a una alumna mejicana. La fase de profundización consistió en 16 entrevistas abiertas en profundidad, 6 profesores, 6 alumnos y 4 padres seleccionados en función de su participación en los programas de compensatoria y diversificación curricular. En total han sido 22 entrevistas abiertas en profundidad ( 9 de alumnado; 8 de profesorado y 6 de familias). Respecto a las familias, las entrevistas se han realizado con 4 madres, 1 padre y 1 conjunta de padre y madre.

\section{Instrumento}

Se ha utilizado la entrevista individual en profundidad como "...técnica de investigación intensiva para profundizar en aquellos aspectos más teóricos y globales que constituyen el discurso especializado (ideológico y profesional) sobre un tema y los fundamentos en que éste se apoya" (Báez, 2007, p. 95).

Siguiendo la clasificación de Ruiz Olabuénaga (2007), las entrevistas han sido individuales, holísticas y no directivas. La duración de las entrevistas osciló entre 50 minutos y $1 \mathrm{~h}$. y 20 minutos. Las entrevistas se han realizado en base a un guión previo muy general de forma que no se perdiera el hilo conductor de los temas considerados relevantes en base a los objetivos de investigación pero que, al mismo tiempo, permitiera una apertura de forma que pudiera aparecer el máximo de información que la persona entrevistada considerara relevante en su experiencia como persona $(y$ madre, padre, alumno y profesor). Se comenzaban con la explicación de los motivos de la investigación, pasando a plantear la entrevista como una conversación sin prisas en la que el interés del investigador se centra en la experiencia y en las explicaciones que proporciona el entrevistado. La posición del investigador ha sido de "no saber", de intentar comprender ya que como dice Ibáñez (1979, p. 308) "no saber es requisito indispensable para la escucha. El que sabe no tiene nada que escuchar. Por grande que sea el conocimiento previo que el investigador posea sobre un tema determinado, no sabe. Y no sabe porque de lo que ha de saber es del texto que allí habrá de producirse. Y si no se ha producido, aún no sabe". De ese modo las preguntas no eran planteadas al modo de cuestiones preestablecidas para todos los entrevistados sino ajustadas a cada experiencia individual intentando que las nuevas preguntas espontáneas que surgían -fruto de la curiosidad y del intento de comprender- no criticaran la actuación de la persona. Las tablas 1 y 2 presentan las guías de las entrevistas en las dos fases.

\section{Procedimiento}

La selección de los informantes se hizo de forma que se incrementara el grado de diversidad de mismos y su pertinencia y relevancia para extraer información acerca de los objetivos de investigación. Las primeras entrevistas tuvieron un carácter exploratorio por lo que los participantes estaban relacionados con diversos

Tabla 1. Guía de entrevista de la fase de exploración

Explicación general acerca del estudio y se dan instrucciones de que se puede hablar de cualquier cosa que se considere interesante en relación al tema

Guía de entrevista para padres y madres

Llevar la conversación y la indagación en torno a:

Nivel familiar/nivel personal: Relación con el adolescente, opiniones y creencias acerca del cambio adolescente y al cambio personal y familiar que implica.

Nivel escolar/instituto: Visión general que la familia tiene del instituto y del sistema educativo. Relaciones de los padres con el profesorado del hijo/a. Opiniones y creencias respecto a las normas del centro.

Nivel social / iguales: Visión acerca de la vida extraescolar del hijo/a.

¿Cómo se lleva con el grupo de amigos/as fuera del centro?

Guía de Preguntas para el profesorado

Experiencia personal en el ejercicio de la docencia:

- Relaciones con el alumnado de la ESO.

- Expectativas y creencias profesionales.

- Deseos, aspiraciones y frustraciones en el ejercicio docente.

- Adaptación a los cambios.

Guía de Preguntas para el alumnado

- Relación con adultos (padres, profesores, etc.)

- Relación con los iguales (compañeros, amigos, etc.)

- Motivación y percepciones acerca del instituto. Experiencias y sentimientos.

- Diferencias instituto / familia.

- Percepción de la influencia educativa del instituto.

- Deseos y expectativas respecto a la educación 
Tabla 2. Guía de entrevista utilizada durante la fase de profundización

Explicación general acerca del estudio y se dan instrucciones de que se puede hablar de cualquier cosa que se considere interesante en relación al tema

Guía de Preguntas

Escolaridad. Escolaridad en primaria y figura del profesor. Diferencias y semejanzas entre PDC y compensatoria y grupos ordinarios. Diferencias y semejanzas con cursos anteriores. Sentimientos asociados a estar en dichos grupos. Valoración del programa.

Figura del profesor: Aspectos considerados esenciales para un profesor. Figuras de profesores de otros niveles educativos que hayan considerado significativos.

Autenticidad relacional: Intentar que detallen los aspectos de la relación educativa constructiva. Qué creen que más les ha ayudado y por qué. Preguntar por si consideran que su relación se establece porque son profesores o si creen que va más allá.

Exploración acerca de empatía: Comprensión, apoyo, confianza, seguridad y de su relación con la influencia en los estudios.

Guía: Efectos de la acción del profesorado. Respeto por las normas. Realización de tareas escolares.

Dotación de sentido: Motivación para estudiar. Conciencia de lo que hacen y para qué. Concordancia con los objetivos propios. Conexión con lo que les gustaría hacer.

Socialización: Relaciones en el aula, en el centro y en la vida.

Cambios percibidos: en sí mismo, en el alumno/a, en el hijo/a.

Efectividad/utilidad: para qué cree que le ha servido. Para qué cree que le servirá en un futuro. En relación con su familia, con los amigos.

centros de secundaria de Castellón y provincia. A partir de la octava entrevista, el grupo de informantes se seleccionó del alumnado, profesorado y familias directamente relacionados con los programas PACG y PDC del IES Álvaro Falomir d'Almassora (Castellón).

El análisis y la transformación de los datos se ha realizado mediante la aplicación del método comparativo constante utilizando los datos de todas las entrevistas. Se ha realizado el análisis línea a línea, comparaciones entre incidentes para la obtención de categorías, comparaciones entre categorías y análisis de las relaciones entre categorías. La saturación teórica se ha utilizado como criterio para dejar de entrevistar, finalizando el proceso con la delimitación e integración de la teoría.

\section{Resultados}

Presentamos los resultados del análisis de los datos a partir de las categorías conceptuales que han emergido en la investigación y de sus indicadores y propiedades (Ver Tabla 3), así como de la organización y relaciones de dichas categorías entre sí en función de la categoría central (CC), denominada Pilotaje Educativo, y del proceso social básico (PSB), denominado Nutrición Relacional (Ver Figura 1).

\section{Categoría central: "Pilotaje educativo"}

La categoría central está relacionada con la mayoría de las categorías y propiedades, y lo está en mayor cantidad que otras categorías encontradas. La categoría central se detecta por "la facilidad en relacionarse y conectarse a los distintos tipos de datos de un modo rápido y prolijo, la potencialidad de explicar las distintas variaciones y condiciones que aparecen en los da- tos, así como las implicaciones en el establecimiento de una teoría formal integrada y fundamentada en los datos. Su aplicación en el análisis de los datos provoca una mayor profundización e implicación con el estudio de los procesos sobre los que el investigador está trabajando (Carrero, 1998). El Pilotaje Educativo es nuestra categoría central ya que por una parte tiene como antecedente inmediato la categoría de encuentro mediante la cual se vinculan e implican afectivamente profesor y alumno en el proceso educativo, y por otra parte contribuye al proceso social básico de nutrición relacional desde el ámbito educativo y permite el desarrollo personal hacia el desarrollo del ser. Como punto de inflexión del proceso de cambio y desarrollo hemos encontrado el paso de la individuación reactiva a la individuación socializada del alumnado.

El pilotaje presenta, como indicadores, las actuaciones del profesorado que transmiten al alumnado aceptación, reconocimiento, confianza, protección, respeto, autenticidad y que dotan de sentido el quehacer educativo. Estas acciones se traducen en la forma en que el profesor organiza y conduce el grupo, en las pautas y normas que establece y en el ajuste de su acción educativa al momento de desarrollo y conocimiento del alumno sobre el que ejerce una acción orientadora. Dichos indicadores se han agrupado en las categorías de sostén afectivo y guía.

\section{Categoría: "Encuentro"}

El encuentro viene indicado por la vinculación afectiva profesor-alumno, por su reconocimiento, aceptación mutua, congeniar, empatía y por el desarrollo de la comunicación que supone un incremento del compromiso y de la implicación de ambas partes en la tarea educativa. La posibilidad de encuentro radica en la autenticidad de la relación propuesta y en la aceptación 
Tabla 3. Categorías, indicadores y propiedades

Categoría

Encuentro

Pilotaje (Estar encima)

Categoría central

(CC)

Individuación reactiva

Individuación socializada

\section{Indicadores y propiedades}

Momento de vinculación afectiva positiva.

\section{Autenticidad relacional:}

Congeniar, empatía, desarrollo de la comunicación.

Reconocimiento y aceptación mutua.

Vinculación afectiva. Afiliación.

\section{Condiciones que favorecen el encuentro:}

Oportunidades del medio.

Momento adecuado.

Esperanza de éxito.

Legitimidad .

Características personales del profesor y del alumno: Autenticidad

\section{Consecuencias:}

Incrementa el compromiso y la implicación emocional en la tarea conjunta.

Despliegue de esperanza.

Conciencia de la existencia de nuevas posibilidades relacionales. Desarrollo de apertura personal.

Contribuye a la nutrición relacional desde el ejercicio docente mediante:

Responsabilidad y cuidado.

Planificación.

Transmisión de normas y valores.

Correcciones, indicaciones y exigencias.

Preocupación por el alumnado. Dedicación e implicación emocional.

\section{Propiedades:}

Canaliza, orienta y vuelve viable el desarrollo.

Proporciona referencias. Transmite pautas, normas y valores.

Proporciona habilidades y técnicas.

Ajusta la realidad y capacidades personales.

Favorece la integración social.

Favorece la autoexpresión.

\section{Componentes:}

Sostén afectivo:

Aceptación

Reconocimiento.

Confianza.

Protección.

Autenticidad.

Respeto.

Sentido.

Guía:

Organización y conducción del grupo

Establecimiento de pautas y normas de conducta.

Ajuste de la transmisión de contenidos curriculares. Adecuación curricular.

Desarrollo de capacidades, habilidades y técnicas. Orientación personal del alumnado.

Percepción de la situación escolar como ataque al propio valor.

Desconfianza en sus posibilidades de aprendizaje escolar. Labilidad emocional.

Autoafirmación en el rechazo de las propuestas educativas del instituto.

Desgana, apatía, escaso esfuerzo.

Desarrollo del nivel de conciencia.

Desarrollo reflexividad, emergencia del "darse cuenta" del propio cambio.

Desarrollo del autorrespeto y la propia valoración.

Comprensión de los propios intereses y planificación del futuro en función de los mismos.

Desarrollo de la agentividad.

Asunción de responsabilidades.

Desarrollo del realismo y de la autonomía.

Internalización de normas y valores proporcionadas por el profesorado.

Mayor dedicación y esfuerzo hacia el estudio.

(Continúa en pág. siguiente) 
Tabla 3. Categorías, indicadores y propiedades (continuación)

\section{Categoría}

Nutrición Relacional

Proceso Social Básico

(PSB)

Desarrollo de ser en contextos educativos

\section{Indicadores y propiedades}

\section{Ocurre:}

En todos los ámbitos de la experiencia humana.

En educación se da a través del pilotaje.

En educación es una relación asimétrica.

\section{Implica:}

Autenticidad relacional.

Respuesta sensible.

Reconocimiento valor y dignidad del otro.

\section{Efectos:}

Desarrollo personal: físico, psíquico y social.

Desarrollo de la autocomprensión.

En educación favorece el cambio de individuación reactiva a individuación socializada.

Desarrollo de la identidad: autorreconocimiento.

Conexión con uno mismo. Desarrollo de la autenticidad.

Desarrollo de la confianza.

Sentido de dignidad y consciencia del propio valor.

Desarrollo de las aspiraciones personales.

Autoexpresión.

Orientación realista hacia el futuro.

Despliegue de un proyecto personal de futuro con sentido.

Fig. 1. Relaciones entre las categorías encontradas

\section{NUTRICIÓN RELACIONAL}

\section{DESARROLLO DEL SER EN CONTEXTOS EDUCATIVOS \\ Desarrollo del alumnado a partir del pilotaje educativo}

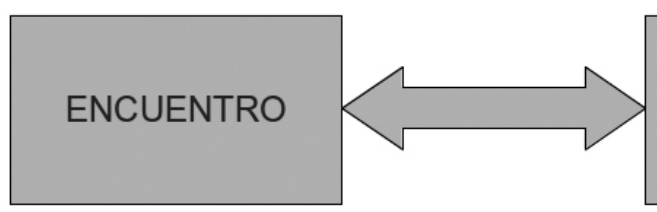

PILOTAJE
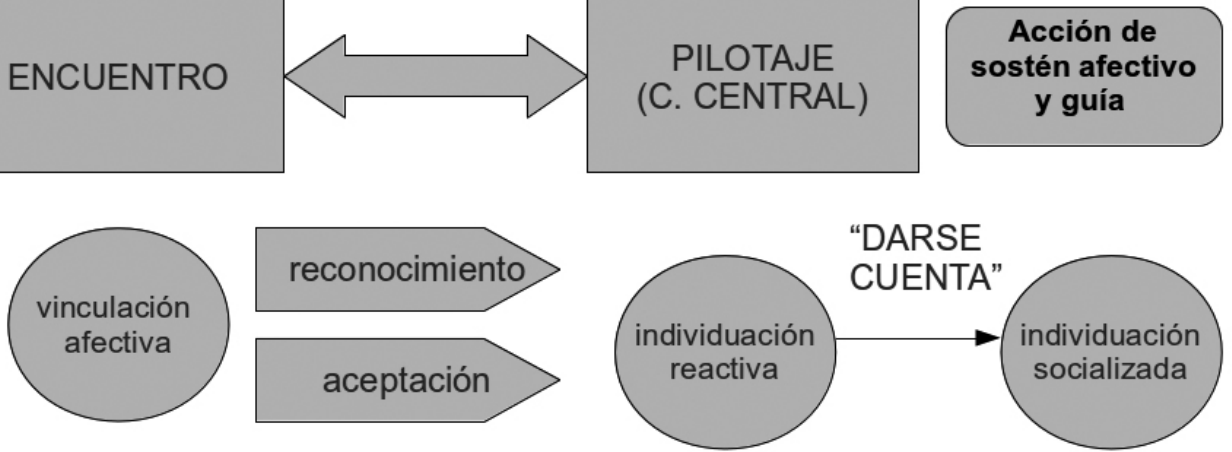

"DARSE

\section{CUENTA"}

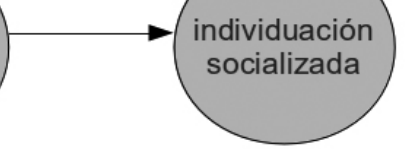

de la misma. La apertura hacia el conocimiento del otro es a la vez causa y consecuencia del encuentro. Las condiciones que favorecen el encuentro son las oportunidades que brinda el medio (en nuestro caso sistema educativo), que el momento sea el adecuado, la esperanza de éxito, el reconocimiento de la legitimidad del sistema educativo y las características personales del profesor y del alumno.

\section{Categoría: "Individuación reactiva" e "Individuación socializada"}

Las categorías de individuación reactiva y de individuación socializada señalan el cambio en el tiempo del alumnado en función de la acción de pilotaje. La individuación reactiva viene indicada por las conductas del alumnado que tienden a autoafirmarse de una 
forma poco productiva o a señalar la carencia de interés y motivación por la propuesta educativa: desgana, apatía, escaso interés, rechazo a las propuestas del instituto a los que subyace una desconfianza en las propias posibilidades de éxito en el seno del sistema educativo.

La categoría de individuación socializada indica un cambio derivado de la restitución de la autoconfianza y de la confianza en el profesorado que pilota el proceso educativo. Esta categoría viene indicada por el desarrollo de la autocomprensión, de la autonomía, de la motivación y de la asunción de responsabilidades de una forma realista.

\section{Proceso Social Básico: "Nutrición relacional"}

La categoría de nutrición relacional, como proceso básico que es, no está saturada en la investigación ya que es un proceso que ocurre en todos los ámbitos de la experiencia humana y no únicamente en el educativo. El proceso se infiere a partir del cambio y desarrollo personal, tanto físico como psíquico y social, que mayor grado de autocomprensión y madurez que supone el paso de la individuación reactiva a individuación socializada.

La importancia del proceso social básico se debe a que "...conforma el objetivo de la generación de teoría en el Grounded Theory. Además de recoger la mayor variabilidad de los distintos patrones observados, informan de los cambios y la evolución de los problemas objeto de estudio. Por ello, son parte fundamental en la integración de teoría y en la generación de teoría formal". (Carrero, 1998).

La nutrición relacional como PSB está caracterizado por una categoría (propiedad del PSB) que se ha referido como "desarrollo del ser". Los indicadores más relevantes de esta categoría son: el desarrollo de la identidad a partir de la conexión con uno mismo, desarrollo de la confianza en las propias posibilidades, la autenticidad, el despliegue realista de las aspiraciones personales que apuntan a la autoexpresión y la concreción del propio proyecto de futuro.

\section{Discusión}

Los resultados de la investigación permiten evidenciar cómo el proceso de socialización, aprendizaje y desarrollo del adolescente se ve altamente potenciado por el proceso de nutrición relacional desde el desarrollo de vínculos afectivos significativos. En el contexto educativo, la nutrición relacional se impulsa desde la vinculación con alguno o algunos profesores a partir del encuentro. En secundaria el ejercicio del pilotaje, entendido como una relación de apoyo y acompañamiento, contribuye a facilitar el desarrollo del ser en un momento de riesgo de "confusión de identidad"
(Erickson, 2000) como es la adolescencia y en el que el adolescente se abre al mundo y tiene la tarea de definir la propia identidad.

La propuesta de investigación realizada permite generar una teoría sobre el pilotaje educativo desde los procesos de nutrición relacional a partir de cinco proposiciones teóricas fundamentadas en el área sustantiva de análisis. Dichas proposiciones se explican a continuación conformando el núcleo de las contribuciones teóricas realizadas al estudio de la relación profesoralumno en centros educativos (ver figura 1).

\section{El Proceso Nutricional Relacional en contextos educativos}

El concepto de nutrición relacional ha sido acuñado por Linares $(2002$; 2006) como amor complejo. En educación nuestros datos presentan algunas diferencias en cuanto a los componentes a través de los que actúa y sugieren su acción en una doble dimensión:

- Una dimensión interior en la que se fomenta la confianza en las propias posibilidades y la toma de conciencia de las aspiraciones, deseos y limitaciones que, más allá, del concepto de autoestima, supone un auténtico sentirse como persona valiosa en sí misma. Esta dimensión se corresponde con la acción de sostén afectivo del pilotaje.

- Una dimensión exterior, de relación con el mundo, que potencia el desarrollo de habilidades y conocimientos para la relación con el medio en el despliegue de las capacidades. Esta dimensión se corresponde con la acción de la guía del pilotaje.

Las dimensiones de la nutrición relacional poseen un alto grado de interdependencia que conlleva que el desarrollo de una sin la otra merme la plenitud del desarrollo. El centro del proceso de nutrición relacional es la confirmación de la persona como única y con gran potencial de desarrollo que se produce a través de interacciones significativas positivas con el otro. El sentimiento de aceptación, y la conciencia de valor personal, permite a la persona reconocerse en sus aspiraciones como ser con inmensas posibilidades de desarrollo futuro y autorrealización, facilitando de ese modo su proceso de autoexpresión.

Aunque la nutrición relacional es un proceso esencialmente positivo, se hace más visible a partir de las consecuencias que implica la carencia de la misma debido a los efectos dramáticos que conlleva, especialmente en los primeros años de vida.

En el sistema educativo, la nutrición relacional está directamente relacionada con la acción del profesorado que se plantea el encuentro como reto, el ejercicio del pilotaje como centro de su trabajo y el desarrollo pleno del alumnado como objetivo último dentro del proceso educativo. Sin estos tres aspectos, la relación queda 
vacía, poco significativa y en el mejor de los casos da lugar a un proceso de mera instrucción.

\section{El desarrollo del ser en el marco educativo}

Pero más allá del cambio de individuación reactiva a individuación socializada, los indicadores que se agrupan en torno a la categoría de desarrollo del ser, nos permiten vislumbrar un proceso más amplio que la transición adolescente, que hemos denominado desarrollo del ser por coincidir en gran medida con las ideas de la psicología del ser de Maslow (1972) y de la corriente de psicología humanista-existencial.

El proceso de expresión del ser constituye la fuerza motivacional de la expresión vital de ser en los distintos ámbitos de la experiencia personal (Carrero y Ruiz, 2009). Desde este punto de vista, el proceso de desarrollo consiste en una progresiva actualización del potencial humano. Actualización que se produce necesariamente en un contexto social determinado, en relación con los demás. De este modo, las fuentes de nutrición relacional que permiten la actualización del potencial humano son siempre las personas significativas y lo ejercen principalmente mediante la vinculación afectiva positiva que les permite proporcionar la confianza y seguridad imprescindible para lanzarse a la exploración tanto del mundo interior como del mundo exterior.

La tendencia o el impulso que toda persona tiene a actualizar dicho potencial, aún en las circunstancias más adversas, designado en la psicología humanista como autorrealización, tendencia actualizadora, plena humanidad, o como procesos de autoexpresión, tal como afirma Carrero (2007), “... sólo pueden ser entendidos desde el vínculo necesario que la dimensión vocacional, como potencialidad innata del ser humano de vivirse en plenitud, adopta en los distintos niveles de conciencia de ser, es decir, desde donde la persona se reconoce como "ser" (procesos de construcción del yo o estadios del yo)".

Durante la adolescencia cobra especial fuerza la definición de identidad como tarea principal del desarrollo del ser, de forma que el adolescente pueda convertirse en adulto y guiar la propia vida. Las cuestiones alrededor del sentido de la vida y de quién soy están presentes o latentes en esta fase. La exigencia de independencia, los signos externos de identificación como la forma de vestir y los adornos constituyen una expresión de dicho proceso de búsqueda de identidad y de individuación mediante la autoafirmación. Los conflictos con los padres y la adopción de conductas del grupo con el que se identifican forma parte del mismo proceso. Es un momento en el que se abre al mundo, en el que quiere probarlo todo y conocerlo todo fascinado por las nuevas posibilidades que percibe en el mundo adulto. La apertura al mundo supone el paso de un mundo conocido y hasta cierto punto previsible hacia otro que, por desconocido, se muestra como excitante y lleno de novedades.

\section{Sentido vital: la apertura desde la confusión}

No obstante también es un momento lleno de confusión, confusión acerca de quién se es y que está muy influida por las exigencias que le plantean la familia, y la sociedad. Confusión interna que requiere del pilotaje para que pueda ir encontrándose como persona. En la medida en que el entorno proporcione estabilidad, claridad, sostén afectivo y guía el proceso se verá favorecido. En el caso en que la confusión social sea alta, los valores y roles que se transmiten estén invertidos o la diversidad de modelos propuestos y las normas y pautas de conducta no sean claras, el proceso de definición de identidad corre el riesgo de alargarse o resultar excesivamente complicado. Las provocaciones, las dificultades para interiorización de normas, la apatía, las dificultades para la definición de las propias aspiraciones y las inversiones de rol, apuntan en este sentido. La ambivalencia y la inseguridad del alumnado, las reacciones exageradas que tienden al desafío y a la autoafirmación así como la necesidad de protección que muchas veces manifiestan señalan el aspecto de la confusión que algunas o muchas veces se trasmite también a los adultos y en ausencia de la función de guía se extiende por el aula o el instituto.

La confusión se produce en la intersección entre el proceso de apertura al mundo y las nuevas necesidades debidas al crecimiento y a las presiones sociales. Es una etapa en la que el adolescente necesita autoafirmarse como ser independiente y valioso aunque sea de forma reactiva. Momento que puede ser contemplado como paradójico: se necesita el sostén afectivo y la guía pero se está hipersensibilizado hacia las posibles intromisiones por lo que únicamente se permitirá el ejercicio del pilotaje a la persona que sea capaz de respetar su dignidad personal.

Este proceso subyacente se expresa muchas veces en los centros educativos en forma de rechazo a las exigencias del quehacer escolar, especialmente cuando entra en contradicción con la definición de la identidad o se percibe que atenta contra la integridad y valor, cosa que puede ocurrir frecuentemente en el alumnado que presenta una historia de dificultades escolares. Dicho alumnado ha tenido una historia personal y escolar que ha disminuido la confianza en sí mismo (por lo que respecta a lo que se le exige desde el sistema educativo) que es percibida muchas veces como un ataque al propio valor como persona. Puede pensar que "no vale" para estudiar, lo que conlleva inseguridad y tristeza; puede rechazar el contexto escolar y desarrollar una individuación reactiva que le permite conservar el propio valor pero a costa de perder en formación y preparación para el futuro. 
En general, el aspecto de "reactividad" implica la existencia de algún tipo de problema que está dificultando la acción educativa. Puede tratarse de una respuesta tanto a la situación familiar, a la escolar o a una combinación de ambas. Además se puede dar un desfase curricular que empuje hacia la irreversibilidad de la situación.

La comprensión de este proceso por parte del profesorado, y la articulación de una propuesta educativa que restituya la dignidad personal y sintonice con el proceso de actualización del potencial del alumnado, permiten la posibilidad del encuentro y de la vinculación así como el posterior ejercicio de pilotaje educativo. De este modo se impulsa el desarrollo personal en un sentido vital y personalizado, en un proyecto de vida que emerge desde la potencialidad y genuinidad de cada alumno.

\section{El encuentro y la vinculación afectiva desde la autenticidad}

El encuentro se puede entender como el momento a partir del cual se produce la vinculación afectiva positiva. El lazo afectivo creado es la base de toda la relación y se establece en función del reconocimiento mutuo como personas valiosas, como seres únicos que, más allá de roles preestablecidos y conductas socialmente impuestas, participan de una relación humana auténtica y real a partir de la autenticidad. El encuentro descansa en último término en la autenticidad de profesor y alumno y confiere un carácter genuino a la relación. La relación establecida de esa forma trasciende los roles de profesor y alumno para situarla en el plano de persona a persona (Rogers, 1978). Supone un reconocimiento del otro que implica necesariamente un respeto por su identidad, su intimidad y decisiones personales. Todo ello permite conectar y desarrollar las aspiraciones personales, restituir la confianza y satisfacer la necesidad de autoexpresión.

La vinculación afectiva relanza el proceso de desarrollo del alumnado y permite que las tareas cobren sentido dentro de un proyecto personal, vital y realista de futuro. El encuentro incrementa el grado de compromiso de los participantes y su implicación emocional en el desarrollo de la tarea propuesta.

A partir del encuentro la relación educativa se vuelve significativa y posibilita realización de los esfuerzos necesarios requeridos por el pilotaje para potenciar el desarrollo personal.

El encuentro emerge como centro del reto educativo al permitir integrar los fines últimos de la educación, en un proyecto conjunto profesor-alumno, que actualiza el potencial de ambos en el trabajo cotidiano. Desde esta re-significación se potencia el sentido de "ser educador", confirmándolos como seres humanos plenos y valiosos e impulsando la esencia de la motivación intrínseca del trabajo educativo al poner en juego la totalidad del ser del profesor en la interacción, proporcionando al alumnado un contacto con la plena humanidad, el sentido educativo y la pasión vital (Vaquer, Carrero y García-Bacete, 2011).

\section{Pilotaje educativo: sostén afectivo y guía como desarrollo del encuentro}

Hemos tomado el término de pilotaje de la tradición pedagógica francesa a través de Évéquoz (1990), aunque el "código en vivo" que apareció en las entrevistas fue el de "estar encima". Se define esta categoría como la relación significativa y auténtica que se deriva del encuentro, de la aceptación mutua y del reconocimiento personal. El pilotaje implica el desarrollo de la relación establecida con el encuentro mediante el ejercicio de la acción docente. Supone confirmar las expectativas suscitadas en el alumnado mediante el apoyo afectivo y la guía en el proceso de desarrollo y aprendizaje. Proporciona de ese modo la credibilidad necesaria para que las exigencias que formula el adulto no sean percibidas de forma negativa sino como expresiones de atención y preocupación.

Así pues, el pilotaje se refiere a un tipo específico de relación profesor-alumno capaz de impulsar el desarrollo del alumnado de una forma potente así como de restaurar posibles aspectos "dañados" de la persona y/o compensar posibles carencias educativas debido a su conexión con el proceso de nutrición relacional.

En el caso del docente el pilotaje es básico para que se produzca el correcto funcionamiento del proceso educativo ya que corresponde al docente dirigir, guiar, aconsejar, orientar y organizar el proceso educativo del alumnado de su aula. Se puede decir que es la relación educativa por excelencia. El pilotaje es una función que aunque remite esencialmente a una relación diádica, en el trabajo educativo también posee un componente de dirección grupal por lo que se puede hablar de un pilotaje grupal o de aula. La mayor parte del trabajo educativo en el contexto escolar se produce en grupo, por tanto, no es suficiente con establecer una buena relación de pilotaje individual, hay que liderar y dirigir un grupo de personas. A veces es el buen establecimiento de relaciones individuales lo que favorece el establecimiento de un buen pilotaje grupal, otras es el trabajo realizado con el grupo el que facilita las relaciones individuales. El carácter distintivo del pilotaje de aula viene determinado por la conducción y guía del grupo. Se trata de un verdadero liderazgo grupal constructivo que aúna y organiza el proceso educativo de todos los miembros del grupo, potenciando los efectos de la nutrición relacional al aprovechar la fuerza positiva del grupo, especialmente en lo que se refiere a socialización, normatividad, colaboración y solidaridad, dando lugar al establecimiento de una visión compartida y cohesión grupal que desemboca en un clima de grupo propicio para el trabajo educativo. 
El pilotaje contribuye al proceso de nutrición relacional desde el contexto educativo, desde los componentes de sostén afectivo y guía, que necesariamente han de funcionar de forma integrada. Esta estructura dual, sostén afectivo y guía, entronca con dos tradiciones bien establecidas tanto en la educación familiar como en la educación escolar: las dimensiones fundamentales propuestas por Rollins y Thomas (1979) en los estudios sobre parentalidad competente, apoyo parental e intentos de control, y los dos componentes de la conducta docente eficaz propuestos por Dunkin y Biddle (1974), amabilidad (warmth) y directividad (directivity); de la misma forma, García Bacete, Monjas, Ferrà, Sánchiz, Marande y Muñoz Tinoco (2011), han señalado que los alumnos desde los primeros años de educación primaria perciben la relación profesor-alumnado en términos de proximidad y control.

Sostén afectivo. El sostén afectivo es una condición necesaria pero no suficiente para el desarrollo que permite tener unas condiciones de partida afectivas que lo facilitan. El sostén afectivo posee un carácter básico, de condición imprescindible para aprovechar al máximo el potencial de las actividades que se proponen desde la función de guía. Constituye el requisito sin el cual las orientaciones y actividades que se proponen a la persona aparecen como vacías y sin sentido.

El sostén afectivo se transmite a través de la actuación general del educador en su actuación docente cotidiana, tanto verbal como no verbal, y confirma o defrauda las expectativas afectivas que el alumnado se ha creado en función de sus experiencias anteriores. Por tanto, el grado requerido de sostén afectivo proporcionado por el educador depende de las experiencias previas del alumno, siendo mayor la exigencia y más difícil de proporcionar en situaciones en las que el alumno ha desarrollado desconfianza y reserva ante la actuación educativa.

Desde la autenticidad del profesor, la aceptación y el reconocimiento del alumno, le transmiten a éste un apoyo por ser él mismo, con independencia de otras consideraciones de tipo más funcional como el producto de las tareas escolares, el aspecto o la procedencia social. También se transmite confianza y protección. Se añade así, a la conciencia del propio valor, la seguridad, la tranquilidad y el sosiego. La confianza supone tanto tener confianza en sí mismo como en los demás. Permite aceptar la guía, y especialmente los aspectos de ésta que suponen frustración.

Guía. La guía constituye el aspecto más pragmático de la relación de nutrición relacional. Se da a partir de las interacciones que se entablan alrededor de los contenidos y/o funciones propios de cada contexto. La guía canaliza y orienta el desarrollo realizando una función de ajuste entre exigencias de la realidad y posibilidades de éxito de la persona; proporciona viabilidad al proceso de desarrollo de la mejor forma posible.

Favorece la adaptación y la integración social mediante la transmisión cultural de las pautas, normas y valores, así como la adquisición y desarrollo de habilidades, capacidades y técnicas. Impulsa hacia el futuro favoreciendo los procesos de autoexpresión de forma realista y hasta cierto punto planificados. La guía implica proporcionar referencias para orientarse en el mapa del crecimiento afectivo. La claridad, solidez y coherencia de pautas, normas y valores favorecen el desarrollo y la socialización mediante el establecimiento de unas "reglas de juego" que permiten "contener" el torrente de estímulos y afectos con que se encuentra el adolescente.

El dominio de la guía es el terreno práctico en el que se mueven los padres y educadores. Es donde se pone a prueba el sostén afectivo. Las acciones realizadas en el plano pragmático son las que proporcionan, confirman, amplían o interfieren el plano del sostén afectivo.

En el marco educativo, la guía se refiere a las actividades que realiza el docente en su ejercicio profesional, es decir, la organización y conducción del grupo, el establecimiento de pautas y normas de conducta, la transmisión de los contenidos curriculares mediante la adecuación curricular, el desarrollo de capacidades, habilidades y técnicas para finalmente englobarlo todo dentro de un proceso de orientación como persona que guía en la toma de decisiones de futuro. En resumen lo que se podría considerar el trabajo por excelencia del docente o, visto de otro modo, la realización de un excelente trabajo educativo.

\section{Darse cuenta: procesos de individualización consciente}

Como consecuencia de la acción del pilotaje del profesor se potencia el proceso de nutrición relacional y el alumnado se desarrolla como persona. La categoría de individuación socializada supone la constatación por parte de los informadores de la percepción del cambio producido en un "darse cuenta" que se realiza como un "salto cualitativo", una comprensión súbita de haber cambiado. Primero y de forma lenta se va produciendo el cambio y en un determinado momento que se les pregunta por ello caen en la cuenta. De esta forma se produce la paradoja de que un mayor nivel de conciencia se alcanza de forma inconsciente y hay que hacer reflexionar sobre ello para que se den cuenta.

De otro lado, el incremento del nivel de conciencia y la toma de contacto con los propios intereses contribuye a ir disipando la confusión establecida alrededor del proceso de construcción de la propia identidad y a la toma de decisiones de forma más madura y de acuerdo con los propios intereses.

La Individuación Socializada implica un grado de madurez y de relación con los demás que ya no responde únicamente a una reacción ante un medio percibido como hostil sino que supone un aprovechamiento productivo de las oportunidades sociales. También se hubiese podido denominar individualización conscien- 
te, primera madurez, etc., pero el hecho de que se puede establecer muy fácilmente una relación y un diálogo con el alumnado nos inclina a pensar en una mayor facilidad para establecer relaciones sociales.

\section{Directrices futuras e implicaciones prácticas}

Desde el estudio realizado, y teniendo en cuenta el conjunto de hipótesis teóricas obtenidas, se pueden proponer las siguientes recomendaciones a modo de sugerencias para poder dar respuesta a los niveles de complejidad, muchas veces rotulados como conflictos, que se conforman en el seno del centro educativo. Por ello es importante reconocer que el centro del trabajo del profesorado se ubica en la propuesta relacional auténtica como persona con la que se pueden establecer lazos afectivos.

Las conclusiones de este estudio ponen de relieve el alto grado de responsabilidad que tiene el profesorado y la dificultad que supone una buena práctica docente. Debido a ello, se considera necesario profundizar tanto en la investigación de los aspectos afectivos y actitudinales del profesor como en las condiciones que favorecen o interfieren en las posibilidades de encuentro. El profesor se revela como figura central del proceso educativo por lo que hay que potenciar su crecimiento como profesional y como persona.

La exigencia profesional radica en primer lugar en el aspecto personal del profesor que le permite abordar las relaciones humanas de forma auténtica dotando de confianza y sentido la relación educativa. En segundo lugar, en su capacidad técnica para guiar y ajustar el proceso educativo de modo que el desarrollo del potencial del alumnado sea viable en el mundo concreto en que vive. La calidez afectiva, la empatía, la aceptación, la autenticidad, la comunicación, el sentido de las acciones, el ajuste a las necesidades del alumnado, proporcionar esperanza, el respeto a la integridad del ser, etc., han aparecido como elementos centrales de la nutrición relacional y caracterizan -en mayor o menor medida- a las personas que consiguen ejercer el pilotaje tanto individual como de aula. Se desprende de ello la necesidad de investigar acerca de los aspectos personales del profesorado que permiten y amplían las posibilidades de encuentro con el alumnado.

Además de en los aspectos personales del profesorado, las posibilidades de encuentro se ven favorecidas por condiciones externas que dependen de la estructuración del sistema educativo. El momento adecuado y las oportunidades del medio señalan la importancia de ofrecer oportunidades al alumnado en diferentes momentos de su desarrollo como persona. La esperanza de éxito señala la importancia de ajustar el trabajo educativo a las condiciones y necesidades concretas del alumnado.

Después de realizada la presente investigación, podemos afirmar que buena parte de las manifestaciones conflictivas que ocurren en los centros de secunda- ria constituyen una emergencia de las dificultades con que se está encontrando nuestra sociedad respecto a la nutrición relacional de nuestros adolescentes. Bien sea por disfunciones familiares, bien por disfunciones del sistema educativo o de la sociedad, queda la impresión de que una buena parte de los adolescentes actuales no tienen el suficiente o el oportuno apoyo afectivo y, desde luego, la guía adecuada. Aspectos como el abandono, en sus muy diversas formas (entre ellas algunos tipos de permisividad, la sobreprotección, la sobreexigencia...), el maltrato (el empleo de castigos físicos), las contradicciones en las normas y valores, las malas experiencias en el entorno escolar, la escasa sensibilidad del sistema educativo o dificultades organizativas de la institución escolar para responder ante estas problemáticas, etc., constituyen diferentes aspectos de maltrato que convendría investigar.

Queda también la sospecha acerca de la falta de sensibilidad social hacia el desarrollo socio-afectivo en general y hacia las medidas de protección de la infancia y de la juventud y de la gran importancia que para una sociedad tiene el desarrollo afectivo y la socialización de ésta. Sin embargo, la sociedad se escandaliza ante los efectos de esta falta de sensibilidad: accidentes de tráfico, alcoholismo juvenil, botellón, incremento de la problemática de drogas, etc. y trata dichos efectos de forma separada queriendo solucionarlo mediante la introducción de asignaturas o con el incremento del autoritarismo, sin reparar en que una y otra vez no han dado resultado ninguna de dichas soluciones. Los vínculos afectivos que proporcionan seguridad, confianza, sentido, normas, pautas de conducta, sentido al quehacer diario, orientación y saber, parecen ser la mejor prevención y salvaguarda de salud psicológica que podemos proporcionar a las personas $\mathrm{y}$, por ende, contribuir a su pleno desarrollo y a la prevención del malestar social.

\section{Agradecimientos}

La realización de este artículo ha sido posible gracias a la ayuda concedida al segundo autor por la Generalitat Valenciana y referida como Experiencias del Fluir: Propuesta de un Modelo de Desarrollo Óptimo para desarrollar la motivación intrínseca en trabajos vocacionales? (Referencia GV05/118) y a la ayuda de investigación concedida al tercer autor por el Ministerio de Ciencia e Innovación (Proyecto: Rechazo entre Iguales y Dinámica Social del Aula: Una Aproximación Multidisciplinar y Multimetodológica (Referencia PSI2008-00541/PSIC).

\section{Referencias}

Báez, J. (2007). Investigación cualitativa. Madrid: ESIC. Bowlby, J. (1998). El apego. Barcelona: Paidós. 
Carrero, V. E. (1998). Análisis cualitativo de datos: aplicación de la teoría fundamentada ("Grounded Theory") en el ámbito de la innovación organizacional. (Tesis de doctorado, Universidad Jaume I).

Carrero, V. E. (2007). El sentido de "ser". Revista Electrónica de Motivación y Emoción. Consultado en http://reme.uji. es/

Carrero, V. y Ruiz, Y. (2009). La motivación desde los procesos de autoexpresión. XI Congreso Nacional de Psicología Social.

Cava, M. J., Musitu, G. y Murgui, S. (2006). Familia, actitudes hacia la autoridad y violencia escolar. Consultado en http://www.infocop.es/view_article. asp? id=1035\& cat $=38$

Charmaz, K. (2005). Grounded Theory in the $21^{\text {st }}$ century. En N. K. Denzin, y Y. S. Lincoln, The sage handbook of qualitative research. California: Sage Publications.

Dunkin, M. y Biddle, B. (1974). The study of teaching. New York: Holt, Rinehart, \& Winston.

Évéquoz, G. (1990). Y a-t-il un pilote dans la classe? Le problème de la hiérarchie dans un système artificiel. Thérapie Familiale, 11, 407-423.

Erikson, E. H. (2000). El ciclo vital completado. Barcelona: Paidós.

Fernández, M. (2001). Educar en tiempos inciertos. Madrid: Ediciones Morata.

Fernández, M.; Mena, L. y Riviere, J. (2010). Fracaso y abandono escolar en España. Barcelona: Obra Social Fundación "La Caixa".

García Bacete, F. J., Monjas, Mª I., Ferrà, P., Sánchiz, Mª L., Marande, G. y Muñoz Tinoco, $M^{a}$ V. (2011). La percepción de los alumnos rechazados de las relaciones profesor-alumnado en su grupo/aula. En J. M ${ }^{a}$ Román, M. A. Carbonero y J. D. Valdivieso (Comps.), Educación, aprendizaje y desarrollo en una sociedad multicultural (pp. 4755-4771). Madrid: Ediciones de la Asociación Nacional de Psicología y Educación.

Glaser, B. G. y Strauss, A. L. (1967). The Discovery of Grounded Theory. Strategies for Qualitative Research. Hawtorne, NY: Aldine de Gruyter.
Ibáñez, J. (1979). Más allá de la sociología. El grupo de discusión: Técnica y Crítica. Madrid: Siglo XXI.

Linares, J. L. (2002). Del Abuso y Otros Desmanes. El Maltrato Familiar entre la Terapia y el Control. Barcelona: Paidós.

Linares, J. L. (2006). Complex Love as Relational Nurturing: An Integrating Ultramodern Concept. Family Process, 45, 101-115.

Marchesi, A. (2004). ¿Qué será de nosotros los malos alumnos? Madrid: Alianza Editorial.

Marina, J. A. (2006). Introducción. Movilización educativa de la sociedad civil. Consultado en http://www.movilizacioneducativa.net/introduccion.asp

Marrone, M. (2001). La teoría del apego. Un enfoque actual. Madrid: Psimática.

Maslow, A. (1972). El hombre autorrealizado. Hacia una psicología del ser. Barcelona: Kairós.

Prats, J. y Raventós, F. (2005). Los sistemas educativos europeos. ¿Crisis o transformación? Barcelona: Fundación "la Caixa".

Rogers, C. R. (1978). Libertad y creatividad en la educación. Buenos Aires: Paidós.

Ruiz Olabuénaga, J. I. (2007). Metodología de la investigación cualitativa. Bilbao: Universidad de Deusto.

Rollins, B. y Thomas, D. (1979). Parental Support, Power And Control Techniques In The Socialization Of Children. Child Development, 56, 524-528.

Vaquer, A., Carrero, V. y García-Bacete, F. (2011). Autenticidad del profesorado: implicaciones en el desarrollo de competencias docentes. En J. Ma Román, M. A. Carbonero y J. D. Valdivieso (Comps.), Educación, aprendizaje y desarrollo en una sociedad multicultural (pp. 7021-7035). Madrid: Ediciones de la Asociación Nacional de Psicología y Educación.

Wentzel, K. R. (2009). Students' relationships with teachers as motivational contexts. In K. R. Wentzel \& A. Wigfield (Eds.), Handbook of motivation at school (pp. 301-322). New York: Routledge.

Manuscrito Recibido: 28/12/2010

Revisión Recibida: 23/02/2011 Manuscrito Aceptado: 09/05/2011 